\title{
Transfeminismo, decolonialidad y el asunto del conocimiento: algunas inflexiones de los feminismos disidentes contemporáneos
}

\author{
Marta Cabrera ${ }^{1}$ \\ Pontificia Universidad Javeriana ${ }^{2}$ \\ marta.cabrera@javeriana.edu.co \\ Liliana Vargas Monroy ${ }^{3}$ \\ Pontificia Universidad Javeriana ${ }^{4}$ \\ liliana.vargas@javeriana.edu.co \\ Recibido: 4 de octubre de 2013 \\ Aceptado: 23 de enero de 2014
}

\footnotetext{
1 Doctora en Comunicación y Estudios Culturales.

2 Profesora asociada, Facultad de Ciencias Sociales, Departamento de Estudios Culturales.

3 Doctora en Psicología Social.

4 Profesora asociada, Facultad de Psicología.
}

Documento accesible en línea desde la siguiente dirección: http://revistas.javeriana.edu.co

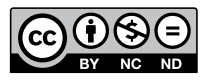




\title{
Transfeminismo, decolonialidad y el asunto del conocimiento: algunas inflexiones de los feminismos disidentes contemporáneos
}

\section{Resumen}

A partir del seguimiento de algunas derivas teóricas de los feminismos contemporáneos, este artículo propone tres disidencias que recogen los que consideramos son algunos de los puntos de inflexión más fuertes en sus debates actuales. Proponemos que desde estas inflexiones se han comenzado a delinear nuevos campos de discusión y nuevas agendas metodológicas y de investigación.

Palabras clave: feminismos disidentes; feminismos de frontera; transfeminismo; feminismos queer; feminismos poscoloniales; feminismo decolonial; ciencia feminista

\section{Transfeminism, Decoloniality and the Problem of Knowledge: Some Inflections of Contemporary Dissident Feminisms}

\begin{abstract}
Based on the monitoring of some theoretical branches of contemporary feminisms, this article proposes three dissents that collect what we believe to be some of the strongest points of inflection in their current debates. We propose that, based on these inflections, new areas of discussion and new methodological and research agendas have begun to be outlined.

Keywords: dissident feminisms; frontier feminisms;transfeminism; queer feminisms; postcolonial feminisms; decolonial feminism; feminist science
\end{abstract}

\section{Transfeminismo, decolonialidade e o assunto do conhecimento: algumas inflexões dos feminismos dissidentes contemporâneos}

\section{Resumo}

A partir do seguimento de algumas derivas teóricas dos feminismos contemporâneos, este artigo propõe três dissidências que recolhem os que acreditamos são alguns dos pontos de inflexão mais fortes nos debates atuais. Propomos que desde estas inflexões começaram-se a delinear novos campos de discussão e novas agendas metodológicas e de pesquisa.

Palavras-chave: feminismos dissidentes; feminismos de fronteira; transfeminismo; feminismos queer; feminismos pós-coloniais; feminismo decolonial; ciência feminista 


\section{Introducción: feminismo, feminismos y el asunto del sujeto}

El feminismo puede ser pensado, más que como un movimiento unificado, como un campo discursivo de acción/actuación, amplio y heterogéneo que no se limita a los grupos u organizaciones denominados feministas, sino que se extiende a otros grupos y espacios de actuación (Alvarez, 1998). Teniendo en cuenta estas características, resulta obvio suponer que los derroteros del feminismo están marcados por cuestionamientos, tensiones y disidencias.

De esta manera, el término disidencia nos interesa aquí en su sentido de 'hacerse aparte', pues nos permite abordar aquellas instancias donde determinados núcleos de discusión teórica y política dentro del feminismo contemporáneo ejercen su crítica y hacen audibles sus voces, interpelando los centros de la teoría feminista. Es en ese sentido que nos referimos a feminismos (en plural) y no a un feminismo monolítico y homogéneo. Se trata en suma, de dar cuenta de la emergencia de una multitud de proyectos y preguntas que responden a tensiones políticas y complejidades teóricas, abriendo nuevas dimensiones en el proceso, siempre inacabado, de definición del feminismo.

Proponemos entonces que uno de los cuestionamientos centrales que desarrollan los feminismos disidentes problematiza la noción de un sujeto (universal) femenino, monolitico y ficticio (en tanto construcción ideológica), que sin embargo fue y continúa siendo funcional para articular una forma de movilización que defiende la adquisición de derechos y el acceso de las mujeres a la esfera pública (Trujillo, 2009 , p. 162). Es claro que la segunda ola del movimiento feminista (así denominada por su discontinuidad histórica con el movimiento sufragista), reabrió y continúa abriendo el debate en torno a la posición social de las mujeres, dando así cabida a una nutrida producción teórica vinculada a prácticas afirmativas del colectivo, por oposición a su 'contrario'. Se trata pues de un discurso identitario asentado sobre la base de las diferencias de género.

Reconociendo la importancia de este movimiento, resulta importante señalar que los feminismos actuales cuestionan, no solamente la universalidad del sujeto moderno (hombre, burgués, blanco, heterosexual), sino también la existencia de un sujeto femenino único (mujer blanca, heterosexual, occidental y por lo general, de clase media o alta). Este sujeto femenino -uniforme y coherente- resulta problemático, pues en terminos generales, desde su pretensión de universalidad, termina ocultando su posición, reproduciendo así lo 
que Donna Haraway (1991) ha denominado el "truco del ojo divino", el cual sustenta un conocimiento en cabeza de un sujeto que parece verlo todo, pero que paradójicamente nunca es visto.

Movimientos como el feminismo negro cuestionan, ya en la década de 1960 , este posicionamiento al denunciar la centralidad discursiva de las mujeres blancas de clase media dentro del feminismo hegemónico. El feminismo lésbico hará lo propio al llamar la atención sobre la "heterosexualidad obligatoria" que parece habitar algunas propuestas feministas (Rich, 1996). A estos se añadirán múltiples cuestionamientos provenientes de identidades fronterizas (chicanas, poscoloniales, transexuales, queer, etc.), desde los que algunas teóricas (Anzaldúa, 1987; Lorde, 1984; Lugones, 2008, entre otras) suman al análisis vectores de opresión de clase, raza, etnia y orientación sexual, argumentando que no se trata de establecer jerarquías entre ellos. Desde este punto de vista, no habría un vector de opresión principal que deba ser asumido por los análisis del feminismo, sino más bien sistemas de opresión múltiples que actúan simultánea y entrecruzadamente (Smith, 1983; Lugones, 2008).

Podriamos decir entonces, que es en el marco del debate igualdad-diferencia que aparecen las fracturas de este sujeto homogéneo. Desde allí, la pretensión de homogeneidad, no se relaciona solamente con el sujeto masculino que promueve sus valores como universales, sino que es claro, habita también al sujeto universal construido por el feminismo, el cual presenta las mismas limitaciones con respecto a otras posiciones identitarias. Las consecuencias de este debate para la teoría y práctica feminista son inmensas e implicarán en suma, que la diferencia ya no estará solamente localizada entre identidades, sino dentro de ellas (Butler, 1989). En otras palabras, el feminismo se desplazará de la diferencia de géneros (de la década de 1960), hacia las diferencias entre mujeres (de la década de los 80), (Casado, 1999).

Se puede afirmar que hasta la introducción de este último giro, -que algunas teóricas vinculan con una tercera ola del feminismo (Drake, 1997; Lotz, 2003; Pujal 2007; entre otras)-, el feminismo se escindía entre posturas y prácticas organizadas a partir de una oposición, cuyas fronteras no estaban demarcadas de manera nítida (Fuss, 1989), pero que en términos generales, oscilaban entre una posición esencialista y otra relativista (la Mujer - las mujeres).

La posición esencialista implicaría cierto reduccionismo. Como lo pondrán de manifiesto tanto el feminismo como otros movimientos sociales, el hacer parte de una cultura o grupo determinado no implica 
necesariamente participar de todas sus estructuras y significados. Por su parte, la corriente antiesencialista correria también sus propios riesgos al considerar, por ejemplo, las categorias esenciales como si fuesen "inmediatamente aparentes y naturalmente transparentes" (Fuss, 1989, p. 21). En otras palabras, la postura antiesencialista vería al esencialismo como si fuese una "teoría estable que funciona siempre en un nivel reaccionario y que escapa al cambio" (Fuss, 1989, p. xii).

Para escapar del binarismo esencialismo-antiesencialismo, Diana Fuss (1989) sugiere la posibilidad de pensar la categoria mujeres como una unidad lingüistica, conceptualmente heterogénea, pero que semánticamente marca una colectividad. Teresa de Lauretis por su parte, invita al pensamiento crítico a negarse a ocupar cualquiera de los polos de la ecuación y desanclar más bien este binario del mundo de la crítica feminista, para reconectarlo con su contexto social y discursivo (1999). Otra noción de utilidad aquí es aquella que repiensa ese sujeto no como hablado simplemente por los discursos, sino provisto de agencia, es decir, activo y con capacidad de actuar como intermediario. La posibilidad de la mediación aboga por una mayor responsabilidad y conocimiento del propio lugar de enunciación, más allá de los planteamientos modernos o las posiciones radicalmente relativistas.

Donna Haraway (1991) vincula estas preocupaciones politicas y epistemológicas con la noción de políticas de la localización (politics of location $)^{5}$. En términos generales, las políticas de la localización introducen las nociones de experiencia y conocimiento en relación con las condiciones de producción del conocimiento y advierten que la relación de estos elementos está fraguada a partir de la "historia, la contingencia y la lucha" (Mani, 1992). De esta forma, las politicas de la localización resisten la construcción de posiciones homogéneas y se comprometen con la exploración de conocimientos múltiples, móviles y relacionales, implican el paso de un sujeto homogéneo y mítico a una agencia en constante proceso de construcción y deconstrucción que adquiere así significado en la praxis y en los márgenes.

Elena Casado presenta una útil tipología de las posiciones que hemos señalado: en primer lugar estarian las visualizaciones explícitamente multidimensionales, entendidas como aquellas fundadas sobre las sedimentaciones de diferentes formas de dominación, que van produciendo sus propias categorizaciones, como en el caso de formulaciones como el "capitalismo racialmente estructurado" (Bhavnani

5 Hay un número de formulaciones del término dentro de las cuales se encuentran la de Rich (1986), hooks (1990), Mohanty (1992), entre otras. 
y Coulson), el "feminismo del tercer mundo" (Moraga y Smith) y las "clases político sexuales" (Sofolius). Las visualizaciones viajeras o de tránsito insisten en el carácter móvil de la identidad y la subjetividad y tienen en cuenta contextos de globalización y migración, como es el caso del "world traveller" (Lugones), el peregrino (Clifford; Bauman) y la subjetividad nómada (Braidotti). Por último, las visualizaciones fronterizas aluden a identidades basadas en la diferencia y que se construyen desde los márgenes de los discursos homogeneizadores, como es caso de bell hooks al describir el movimiento "de los márgenes hacia el centro", o el "desplazamiento del centro a los márgenes" del sujeto poscolonial de Gayatri Spivak, o la "casa de la diferencia" de Audre Lorde (Casado, 1999, pp. 86-87).

En suma, las políticas de la localización dan cuenta de las complejidades de la producción de conocimiento y resultan invaluables para efectuar una serie de desplazamientos, como los que propone Beatriz Preciado:

1. [de] las teorias y movimientos anti-coloniales hacia una crítica post-colonial; 2. del feminismo hegemónico heterocolonial hacia una crítica de la construcción transversal de la raza, del sexo, del género y de la sexualidad; 3 . de las politicas de las identidades hacia las políticas post-identitarias; de las políticas de los cuerpos hacia las cyborgologias desnaturalizadas (Preciado, s.f.).

De esta manera, y reconociendo la existencia de muy diversas líneas de fuga dentro de los feminismos contemporáneos, nos centraremos aquí en tres desplazamientos particulares, que creemos se ven reflejados en el presente número de Universitas Humanistica. Si bien es claro que no son las únicas derivas que es posible mapear, sí creemos que recogen algunos de los puntos de inflexión más fuertes, dentro de los debates actuales del feminismo, que han comenzado a delinear nuevos campos de discusión, y nuevas agendas metodológicas y de investigación. En suma, proponemos tres zonas de discusión que pretenden problematizar y provocar pensamiento y dialogo en torno a los horizontes del feminismo. 


\section{Del género como categoria de análisis / determinante de desigualdad, al género como dispositivo de captura}

Frente al problema que implican los bebes intersexuales para el ejercicio de la asignación sexual binaria, John Money propone en 1955 el término rol de género (gender role) para describir el conjunto de conductas atribuidas diferencialmente a las mujeres y los varones (Money y Tucker, 1978). La propuesta de Money es que el género es una construcción social (por tanto, con el tratamiento médico y psicológico adecuado, se podría hacer que un hombre se transformara en mujer o viceversa). Bajo esta perspectiva, Money inicia un ejercicio de reconstrucción técnica de los bebés intersexuales que incluirá intervenciones quirúrgicas, hormonales y por supuesto, psicológicas (Preciado, 2011). En la década siguiente, el libro de Robert Stoller Sex and Gender: On the development of masculinity and femininity (1968) desarrolla el concepto de identidad de género, distinguiendo el sexo biológico del género social, es decir, instalando esta diferencia, como una instancia de la oposición naturaleza-cultura.

Unos años después, el texto de Ann Oakley Sex, Gender and Society (1972), se establece como otro antecedente relevante para el discurso sobre género al atribuirle al sexo las diferencias fisiológicas entre hombres y mujeres de una parte y al género, las pautas de comportamiento culturalmente establecidas en el ámbito de lo femenino y lo masculino. Es a partir de estas discusiones que se instala firmemente la separación entre un ámbito biológico (el sexo) y uno social (el género), diferenciación que el feminismo de la segunda ola tomará como una de sus ideas centrales.

En este sentido, a mediados de la década de 1970, Gayle Rubin acuñará la noción de sexo-género, que será clave para el desarrollo de una gran variedad de feminismos (Haraway, 1991). El concepto hace referencia a las formas de relación establecidas entre mujeres y hombres en el seno de una sociedad y define condiciones sociales diferenciadas en razón de los papeles y funciones que les han sido asignadas socialmente y de su posición social como seres subordinados o seres con poder sobre los principales recursos (Rubin, 1996). Esta posición será problematizada posteriormente dadas sus pretensiones universalizantes, las cuales se denunciarán como parte de un ensamblado teórico etnocéntrico e imperializante proveniente de los feminismos europeos y euro-estadounidenses, cuyos esfuerzos por caracterizar a la 'mujer del tercer mundo' dieron lugar frecuentemente, a la reproducción de discursos racistas y colonialistas (Mohanty 1984; 2013). 
Más aún, se señalará cómo la noción sexo-género excluye a las mujeres que no se encuentran en posiciones similares en la institución del parentesco. Como señala Haraway, sería entonces válido preguntar: "¿qué ocurre con la idea del género si grupos enteros de mujeres y de hombres están situados juntos fuera de la institución del parentesco, pero relacionados con el sistema de parentesco de otro grupo dominante?" (1991, p. 244).

En la década de 1980, la categoría de género también es sometida a escrutinio en tanto está basada en una serie de polaridades (masculino/femenino, cultura/naturaleza, etc.), esto aunado a la visibilidad de identidades sexuales transgresoras hará que para la década de 1990 Judith Butler problematice en su libro Gender Trouble la naturalizacion del binarismo de género (femenino/masculino), para sugerir que son más bien los actos performativos, repetitivos (basándose en Austin y Searle) los que modelan y definen al género. Tales actos moldean la noción de existencia de dos géneros organizados, idealizados y deseados en detrimento de otras expresiones. Al decir de Butler (1990), al teorizar que el género es una construcción radicalmente independiente del sexo, el género mismo viene a ser un artificio libre de ataduras, por lo que hombre y masculino podrian significar tanto un cuerpo femenino como uno masculino; mujer y femenino, tanto un cuerpo masculino como uno femenino.

Esta teorización será clave para una serie de disidencias sexuales denominadas queer (marica, raro, extraño) que surgen en los años ochenta como respuesta a las politicas identitarias tanto del feminismo como del movimiento de lesbianas, gays, transexuales y bisexuales. Estos sujetos reivindicarán su 'rareza' como una forma de desafiar tanto la norma heterosexual y binaria del género, como las políticas de la identidad. En 1991, Teresa de Lauretis empleará el término teoría queer para cuestionar la existencia de identidades sexuales monolíticas, usando como punto de partida la crítica a la heterosexualidad obligatoria (Rich, 1986; Wittig, 2006), la crítica a la división privado/ público (Millet, 1969) y la noción foucaltiana de biopolítica. Con esto, la teoría queer entra de lleno en la crítica de las fuerzas normalizadoras de la sexualidad, incluyendo la homosexualidad. Finalmente, es posible registrar que la conceptualización del género es también problematizada en los últimos años a partir de una revisión a las intervenciones que buscan insertar a las mujeres del llamado tercer mundo dentro de los órdenes económicos mundiales desde perspectivas de género. Esta es una acción que los feminismos disidentes comienzan a denunciar como parte de un feminismo neoliberal, ampliamente 
extendido hoy en dia, que trabaja a partir de las agendas de agencias internacionales (Preciado, 2011; Falquet, 2011; Mohanty, 2013).

Esto no implica sin embargo, que la noción de género no sea vigente o resulte inútil para dar cuenta de muchas de las desigualdades presentes en el mundo contemporáneo, pero sí muestra que es necesario tensionar y problematizar el concepto para evitar que este sea útil dentro de un ejercicio de normalización que determine y construya lo que deben ser las mujeres, tal como lo han discutido buena parte de los feminismos contemporáneos.

El trabajo central de Judith Butler a este respecto, nos permite pensar una genealogía de la ontología de género, que señalaría que la categoría de género, situada en contraposición a la noción de sexo resulta ante todo, un aparato de captura. Esta genealogía permite evidenciar, como lo ha planteado Beatriz Preciado (2008), que la categoría género es de fecha reciente, apareciendo como lo hemos mencionado, en los años cincuenta, para permitir una práctica de normalización de cuerpos abyectos (inicialmente, los cuerpos de bebés intersexuales). Lo cual hace posible definirla como un dispositivo (Amigot y Pujal, 2009) que construye tanto lo femenino como lo masculino dentro de una máquina de determinación binaria. Diremos finalmente que el género, al (re)producir a las mujeres dentro de la matriz heterosexual de poder, ha servido para gobernar sus cuerpos, actuando además en espacios tan variados como sus lugares de trabajo o sus relaciones sexuales o afectivas.

\section{De la mujer en la ciencia a la ciencia del feminismo: el problema de la producción de conocimiento en clave feminista}

El recorrido realizado hasta aquí, posibilita afirmar que el desplazamiento desde el problema de la mujer en la ciencia, al problema de una ciencia del feminismo, debe ser comprendido ante todo, a partir del posicionamiento particular de las mujeres tanto dentro del sistema sexo-género, como dentro de los múltiples lugares que aparecen cuando se problematiza el género como el principal elemento articulador del feminismo, ejercicio que hemos caracterizado como un paso de la diferencia de género a la diferencia entre las mujeres. El plantear una ciencia del feminismo implica recoger una serie de reflexiones -situadas-que problematizan la producción del conocimiento desencarnado que hasta ahora ha ocupado el lugar hegemónico (al menos dentro de 
la particular producción de conocimiento en occidente), que a la postre, determinará el ascenso de la ciencia moderna. Esta reflexión, proveniente de diversas discusiones del feminismo contemporáneo (Fox Keller, 1985; Harding, 1986; Anzaldúa, 1987; Haraway, 1988; Sandoval, 1995), alterará puntos neurálgicos en las formas de producir conocimiento (Adan, 2006), permitiendo a algunas autoras hablar de una ciencia sucesora feminista (Harding, 1986). Esta transición, que nos parece una forma de disidencia tal como la hemos concebido en el presente texto, nos permite discutir a continuación algunos de los supuestos centrales de esta ciencia sucesora (Harding, 1986).

En primer término la propuesta implica el desenmascaramiento de una posición que Donna Haraway (1997) -siguiendo a los sociólogos de la ciencia Steve Shapin y Simon Schaffer (1985) - denominará testigo modesto, discutiendo la construcción de un tipo de testimonio que garantiza su objetividad a través de un ejercicio de auto-invisibilidad. Boyle habria propuesto en varios de sus textos, que la modestia, es decir la capacidad de borrar la propia subjetividad en el ejercicio científico, era una virtud necesaria y deseable. La modestia sería entonces una de las virtudes fundadoras de lo que denominamos ciencia moderna. Haraway (1997), calificará esta acción como un ejercicio de ocultamiento y con ironía afirmará que esta 'virtud' garantiza que el testigo modesto sea un "ventrílocuo legítimo y autorizado del mundo de los objetos, sin agregar nada de las propias opiniones, o de su influyente corporeidad" (Haraway, 1997, p. 42).

Esta discusión, desarrollada en el marco del feminismo blanco estadounidense, ha derivado en propuestas como las de conocimientos situados, objetividad fuerte y politicas de la localización, de gran relevancia dentro de las discusiones contemporáneas sobre feminismo y conocimiento (Adan, 2006). Estas propuestas procurarán en suma, desenmascarar la posición de supuesta objetividad desde la que se ha construido el conocimiento científico, que para Haraway se encuentra en el centro del modelo de la ciencia occidental, moderna, europea y masculina que nos acompaña hasta nuestros dias (Haraway, 1997).

La propuesta feminista de un conocimiento situado se aleja entonces del objetivismo que plantea la existencia de verdades universales, así como de las visiones relativistas, apelando más bien a un nuevo tipo de objetividad, una objetividad fuerte (Harding, 1986) que explicita el lugar de la mirada y es producto de conexiones parciales y de conocimientos encarnados, limitados y comprometidos. Esta forma de objetividad está estrechamente conectada con una politica de 
localización (Haraway, 1988) que implica un reconocimiento explícito, de la posición particular que se ocupa al producir conocimiento. Para Haraway, el conocimiento científico solo sería lícito a partir de estas conexiones y de un posicionamiento que se entronca con esta ciencia del sucesor:

[...] una ciencia que ofrece una versión del mundo más adecuada, más rica y mejor, en relación crítica y reflexiva con nuestras prácticas de dominación y con las de otros y con las partes desiguales de privilegio y de opresión que configuran todas las posiciones. (Haraway, 1991, p. 321)

Las contribuciones del feminismo blanco estadounidense a los estudios feministas de la ciencia, si bien son sustanciales, no son las únicas, ni las primeras disidencias rastreables en la producción de un conocimiento posicionado, crítico y significativo y su propuesta entra en diálogo con otras discusiones paralelas en las ciencias sociales contemporáneas. Sin embargo, señalaremos una combinación de características que le da cierta particularidad a la propuesta feminista: en primera instancia, su producción de conocimiento implica un giro reflexivo, donde la forma misma de la escritura revela de manera explicita a quien investiga (Albertin, 2000) y su mirada particular, lo que constituye un cierre al testigo modesto; en segunda instancia, se busca la inclusión de un componente ético político y de cuidado, lo que se ha convertido en uno de los rasgos centrales de muchos feminismos disidentes, finalmente se trata de una deriva crítica que busca hacer explícito un análisis de las relaciones de poder. Los anteriores elementos pueden ser reconocidos también, en algunas propuestas teóricas de la feminista chicana Gloria Anzaldúa

Con el concepto de Auto historia/auto teoría, Anzaldúa (1987) propone una forma de producción de conocimiento que se concreta en un "ensayo personal que teoriza" y en un "trabajo autorreflexivo" que implica "una opción por la justicia social" (Anzaldúa, 2002, p. 32). Esta última característica se vincula con la noción de Las alianzas del mundo zurdo, entendida como una búsqueda de formas de conocimiento vinculadas al cambio social y a la emergencia de un nuevo mundo donde la diferencia pueda tener cabida (Anzaldúa, 1987). La obra de Anzaldúa, -una fuente importante para los feminismos decoloniales, que se discuten a continuación-abogará por el reconocimiento de conocimientos otros, que no tendrían cabida en el canon del conocimien- 
to científico; en este sentido, su deriva es aún más profunda y fuerte que la que ocurre en el marco de los estudios feministas de la ciencia. La definición de conocimiento en la obra de Anzaldúa no se circunscribe al ámbito del conocimiento científico y se asocia más bien a un camino de transformación que ella considera como el prerrequisito y la posibilidad más clara de llegar a incidir en el mundo social. Así, los feminismos de color y decoloniales, en los que se suele ubicar su propuesta, se plantean como feminismos que hacen ante todo, nuevas apuestas epistémicas (Espinosa-Miñoso, 2014).

\section{Del sujeto femenino universal al problema de la geo y corpopolítica en la definición del sujeto del feminismo}

Los feminismos pos y decoloniales, el black feminism, los feminismos de color y tercermundistas marcan una tercera forma de disidencia con respecto al feminismo blanco hegemónico, relacionada con la visibilización de los posicionamientos geo y corpopolíticos de las mujeres, cuestión que reabre y radicaliza la crítica a un sujeto universal femenino, que hemos discutido con anterioridad. Para las teóricas feministas que se posicionan dentro de estas trayectorias, la discusión del feminismo blanco habría dado prevalencia a los análisis de género, soslayando otra gran cantidad de marcas identitarias desde las que se ha definido a las mujeres. Este gesto borraria las posibilidades de un análisis de las condiciones históricas que le dieron origen a una organización social que sostiene estructuras jerárquicas de opresión que no son explicables a partir del género exclusivamente (EspinosaMiñoso, 2014).

Podemos decir que las relaciones entre estos feminismos y los estudios subalternos y pos/decoloniales son múltiples, y que sus discusiones se han alimentado mutuamente. Tales discusiones tienen en común la problematización no solo de la dominación colonial, sino de los legados coloniales que se cristalizan en lo que el teórico peruano Aníbal Quijano (2000a) denomina la colonialidad del poder, un patrón mundial de poder que se asienta en modelos cognitivos clasificatorios que jerarquizan las poblaciones del planeta a partir de la idea de raza, siendo funcionales al ascenso del capitalismo ${ }^{6}$. Como respuesta, el llamado giro decolonial buscaria "un cambio de actitud

Arturo Escobar recoge este y otros trabajos para nominar el Programa Modernidad/Colonialidad, estos "localizan su cuestionamiento en los bordes mismos de los sistemas de pensamiento e investigaciones, hacia la posibilidad de modos de pensamiento no-eurocéntricos” (2003, p. 55). 
en el sujeto práctico y de conocimiento, y luego, la transformación de la idea al proyecto de la de-colonización" (Maldonado-Torres, 2008, p. 159). Tanto el giro de-colonial, como la idea de des-colonización:

[...] encuentran sus raíces en la respuesta visceral de los sujetos conquistados ante la violencia extrema de la conquista, que invalida los conocimientos, formas de ser, y hasta la misma humanidad de los conquistados. Los principios del giro de-colonial y la idea de descolonización se fundan sobre el "grito" de espanto del colonizado ante la transformación de la guerra y la muerte en elementos ordinarios de su mundo de vida, que viene a transformarse, en parte, en mundo de la muerte, o en mundo de la vida a pesar de la muerte. La idea de la de-colonización también expresa duda o escepticismo con respecto al proyecto colonial. (Maldonado-Torres, 2008, p. 159)

Ahora bien, el feminismo local -y hablamos aquí desde nuestras determinaciones corpo y geopoliticas-, ha hecho también extensas revisiones de historias marcadas por el racismo, el heterocentrismo y el apego a categorías de análisis provenientes de los feminismos occidentales, situando reflexiones específicas en torno a temas de raza, militarismo, violencia cotidiana y machismo, entre otros, (Gargallo, 2014, pp. 24-25). Esta revisión resulta particularmente importante ya que implica la tensión de las nociones de género asignadas por el poder hegemónico, que tienen profundas raíces coloniales. Este tensionamiento implica el reconocimiento del género como una construcción social que, sobre la base de la genitalidad, produce cuerpos sexuados que se localizan en un esquema jerárquico donde lo femenino denota inferioridad y otras opciones por fuera de este binario hombre/mujer, o incluso la resistencia a reconocerse en el género asignado, son descartadas. Esto hace posible rastrear rupturas y continuidades entre formas coloniales de dominación que articulan contemporáneamente el racismo, el machismo, el sexismo, etc.

Es en este sentido que el trabajo de María Lugones $(2008,2011)$ problematiza la noción de colonialidad del poder. Lugones (2011) planteará en concreto una lectura interseccional que permite comprender la forma en que género y raza se han intersectado en los diversos procesos de producción de las mujeres, superando la comprensión patriarcal y heterosexual de las disputas por el control del sexo, los recursos y los productos, que Quijano ha propuesto como eje central de lectura de la colonialidad del poder. Para Lugones (2011), Quijano habría aceptado el entendimiento capitalista y eurocentrado del género, 
el cual incluye la adopción/internalización de la dicotomía entre hombres y mujeres como una construcción normativa de lo social, aspecto que quedó entretejido con la historicidad de las relaciones, incluyendo las relaciones intimas (p. 106). En otras palabras, la colonialidad y la colonialidad del género estarían imbricadas en el tejido mismo de las relaciones sociales y sería notoria su impronta en los feminismos hegemónicos, los cuales reproducen estrategias de constitución de otredad (mujeres afrodescendientes, indígenas, lesbianas, obreras, trabajadoras del sexo, campesinas, pobres) que terminan siendo útiles al status quo (Espinosa-Miñoso, 2009, p. 45).

Las revisiones que hemos mencionado se vinculan necesariamente con una propuesta epistemológica de decolonización del conocimiento, capaz de develar la manera en que las representaciones de aquellos construidos como otros, dan cuenta de un contexto histórico particular, que continúa reproduciendo relaciones de desigualdad (Suárez y Hernández, 2008). Ahora bien, la propuesta de un giro decolonial, centrado sobre la cuestión de la colonización como componente constitutivo de la modernidad y la de-colonización como un cambio de la perspectiva y actitud que se encuentra en las prácticas y formas de conocimiento de sujetos colonizados (Maldonado-Torres, 2008,), debe también entrar en conversación con los otros feminismos latinoamericanos.

Sin embargo, como Breny Mendoza (2010) ha señalado, en algunas de las propuestas pos/decoloniales latinoamericanas, ha habido un dialogo más estrecho con las feministas chicanas (Anzaldúa y Sandoval, por ejemplo) que con las mismas latinoamericanas, lo cual no deja de sorprender, teniendo en cuenta que la mayoría de autores que articulan estas discusiones son hombres latinoamericanos, blancos y mestizos, heterosexuales y de clase media. Adicionalmente, como señala Ochy Curiel (2007, p. 93), estos mismos teóricos han "dejado por fuera de su análisis categorías como sexo y sexualidad, limitándose a apuntar a la raza como criterio determinante en la división sexual del trabajo".

En contraste, existen numerosos aportes de mujeres racializadas de América Latina y el Caribe que han puesto en evidencia las secuelas violentas del mestizaje en el continente, las relaciones patriarcales, racistas y sexistas, cuestionando los aspectos culturales que perpetúan la subordinación de las mujeres (Carneiro, 2005; Mena, 2007; Viveros, Rivera y Rodríguez, 2006; Wernerk, 2005). Francesca Gargallo (2014) por su parte, reseña los feminismos indígenas y comunitarios haciendo un recuento detallado de experiencias concretas que 
matizan notablemente la noción (más bien homogénea y monolítica) de colonialidad del poder de Aníbal Quijano.

Después de este recuento, podemos afirmar que un feminismo de inspiración decolonial implica la activación de formas de actuación política, que parten de locaciones específicas, del cuestionamiento permanente al sujeto único y de la consideración de las maneras complejas en las que se imbrican los diferentes sistemas de dominación. Finalmente y como propone Lugones, implica igualmente "aprender unas acerca de las otras, como personas que se resisten a la colonialidad del género" (2011, p. 115), en una nueva geopolítica feminista del "saber y el amar" (p. 117).

\section{Referencias}

Adan, C. (2006). Feminismo y conocimiento. Galicia: Spiralia Ensayo.

Álvarez, S. (1998). Feminismos Latinoamericanos. Estudos Feministas, 2, s.p.

Amigot, P., y Pujal, M. (2009). Una lectura del género como dispositivo de poder. Revista Sociológica. Aproximaciones Al Poder, 70 (20), 115-151.

Albertín, P. (2000). Un estudio etnográfico sobre usuarios(as) de heroina. Conocimiento psicosocial y práctica reflexiva. Tesis Doctoral no publicada. Universidad Autónoma de Barcelona, Barcelona.

Anzaldúa, G. (1987). Borderlands/La Frontera. The new mestiza. San Francisco: Aunt Lute Books.

Anzaldúa, G. (2002). Now let us shift ...the path of conocimiento...inner work, public acts. En Anzaldúa, G. y Keating, A. L. (Eds.). This bridge we call home: Radical visions for transformation (pp. 540-578). London: Routledge.

Anzaldúa, G. y Moraga C. (1981). This bridge we called my back. New York: Kitchen table, women of color press.

Butler, J. (1989) Gender trouble, feminist theory, and psychoanalytic discourse. En Nicholson, L. Feminism/Postmodernism (pp. 324-340). Londres y Nueva York: Routledge,.

Butler, J. (1990). El género en disputa. El feminismo y la subversión de la identidad. Madrid: Paidós.

Carneiro, S. (2005). Ennegrecer el feminismo. Número especial sobre Feminismos disidentes en América Latina y el Caribe. Nouvelle Questions Féministes, 24 (7), 21-26.

Casado, E. (1999). A vueltas con el sujeto del feminismo. Politica y Sociedad, 30, 73-91. 
Coba, L. y Gioconda H. (2013). Nuevas voces feministas en América Latina: continuidades, rupturas, resistencias?. Íconos 45, 17-23.

Curiel, O. (2007), Crítica poscolonial desde las prácticas políticas del feminismo antirracista. Nómadas, 26, 92-101.

Drake, J. (1997). Third wave feminism. Feminist Studies, 23, 7- 30

Dube, S. (2008). Asuntos de la modernidad. Estudios de Asia y África, XLIII (1), 83-109.

De Lauretis, T. (1999). Upping the Anti (sic) in Feminist Theory. En During, S (Ed.). The Cultural Studies Reader (pp. 307-319). New York y Londres: Routledge, Psychology Press

Escobar, A. (2003). Mundos y conocimientos de otro modo. El programa de investigación Modernidad/Colonialidad Latinoamericano. Tabula Rasa, 1, 51-86.

Espinosa-Miñoso, Y. (2009). Etnocentrismo y colonialidad en los feminismos latinoamericanos: complicidades y consolidación de las hegemonías feministas en el espacio transnacional. Revista Venezolana de Estudios de la Mujer, 14 (33), 37-54.

Espinosa-Miñoso, Y. (2014). Una crítica descolonial a la epistemologia feminista crítica. Revista El cotidiano. Género: visiones y debates necesarios, 7- 12. Disponible en: http://www.elcotidianoenlinea.com.mx/beta/numeros.asp

Falquet, J. (2011). Por las buenas o por las malas: las mujeres en la globalización. Bogotá: Universidad Nacional de Colombia, Pontificia Universidad Javeriana, Instituto Pensar.

Fuss, D. (1989). Essentially Speaking: Feminism, Nature \& Difference. New York: Routledge.

Fox Keller, E. (1985). Reflexiones sobre género y ciencia. Valencia: Alfons el Magnanim.

Gargallo, F. (2007, enero-junio). Feminismo Latinoamericano. Revista Venezolana de Estudios de la Mujer, 12, 28.

Gargallo, F. (2014). Feminismos desde Abya Yala. Ideas y proposiciones de las mujeres de 607 pueblos en nuestra América. Ciudad de México: Editorial Corte y Confección.

Haraway, D. (1988). Situated Knowledge: The Science Question in Feminism as a Site of Discourse on the Privilege of Partial Perspective. Feminist Studies, 14 (3), 575-599.

Haraway, D. (1991). Ciencia, cyborgs y mujeres. La reinvención de la naturaleza. Madrid: Cátedra.

Haraway, D. (1997). Testigo Modesto@Segundo Milenio. Hombre_hembra. Conoce Oncoratón "Feminismo y Tecnociencia" Barcelona: UOC. Colección Nuevas Tecnologías y Sociedad. 
Harding, S. (1986). Ciencia y feminismo. Madrid: Morata.

Harding, S. (1998). Is science multicultural? Postcolonialism, feminism and epistemologies. U.S.A.: Indiana University Press.

hooks, b. (1991). Choosing the margin as a space for radical openness. En Yearning (pp. 145-153). London: Turnaround,

Lorde, A. (1984). Sister Outsider. Trumansburg, NY: Crossing Press.

Lugones, M. (2008). Colonialidad y género. Tabula Rasa, 9, 73-101.

Lugones M. (2011). Hacia un feminismo descolonial. La manzana de la discordia, $6(2), 105-119$.

Lugones, M. (2012). Subjetividad esclava, colonialidad de género, marginalidad y presiones múltiples. En Pensando los feminismos en Bolivia. La Paz: Conexión Fondo de Emancipaciones (Serie Foros 2).

Lotz, A. (2003). Comunicating third wave feminism and new social movements. Women and Languaje, 26 (1), 2-21.

Maldonado-Torres, N. (2007). Sobre la colonialidad del ser: contribuciones al desarrollo de un concepto. En Castro-Gómez y Grosfogel (Comps.). El giro decolonial: reflexiones para una diversidad epistémica más allá del capitalismo global (pp. 127-167). Bogotá: Siglo del Hombre, Universidad Central, Instituto Pensar, Pontificia Universidad Javeriana.

Maldonado-Torres, N. (2008). La descolonización y el giro des-colonial, Tabula Rasa, 9, 61-72.

Mani, L. (1992). Multiple mediations: feminist scholarship in the age of multinational reception. En Crowley, $\mathrm{H}$ y Himmelweit, S. (Eds). Knowing women. Feminism and knowledge (pp. 306-322). Cambridge: Polity Press, The Open University.

Mena, L. (2007). Raza, género y espacio: las mujeres negras y mulatas negocian su lugar en la Habana durante la década de 1830. Revista de Estudios Sociales, 26, 73-85.

Mendoza, B. (2010). La epistemología de sur, la colonialidad del género y el feminismo latinoamericano. En Espinosa-Miñoso, Y. Aproximaciones criticas a las prácticas teórico-politicas del feminismo latinoamericano (pp. 19-36). Buenos Aires: En la frontera.

Millet, K. (1969) Sexual Politics. Granada: Granada Publishing.

Mohanty, C. T. (1984). Feminist Scholarship and Colonial Discourses. Boundary 2, 12 (3), 333-358.

Mohanty, C. T. (1992). Feminist encounters: Locating the politics of experience. En M. Barrett y A. Phillips (Eds.). Destabilising theory: Contemporary feminist debates (pp. 74-93). Cambridge: Polity Press. 
Mohanty, C. T. (2013). Conferencia: Instituciones académicas neoliberales y la aproximación política del feminismo sin fronteras. Fundación Universidad Autónoma, Bogotá-Colombia.

Money, J. y Tucker, P. (1978) Asignaturas sexuales, Barcelona: A.T.E,

Oakley, A. (1972). Sex, Gender and Society. London: Temple Smith.

Preciado, B. (2008). Testo Yonki. Madrid: Espasa

Preciado, B. (2011). Cuerpo impropio. Guía de modelos somatopolíticos y de sus posibles usos desviados. Seminario Universidad Internacional de Andalucia. Sevilla, España. Audio disponible en: http://ayp.unia.es/index. php?option $=$ com_content $\&$ task $=$ view $\&$ id $=678 \&$ Itemid $=93$

Preciado, B. (s.f.). Saberes_vampiros@War. Disponible en: http:// biopoliticayestadosdeexcepcion.blogspot.com/2010/08/saberesvampiroswarbeatriz-preciado.html

Probyn, E. (1990). Travels in the postmodern: making sense of the local. En L. J. Nicholson (Ed.). Feminism/postmodernism (pp. 176-187). London: Routledge.

Pujal, M. (2007). El feminismo. Barcelona: Editorial UOC,

Quijano, A (2000a, summer/fall) Colonialidad del poder y clasificación social. Journal of World-Systems Research. Festschrift for Immanuel Wallerstein, part I, VI.2, 342-386.

Quijano, A. (2000b). Coloniality of power and eurocentrism in Latin America. International Sociology, 15 (2), 215-232.

Rich, A. (1986). Notes towards a Politics of Location. Blood, Bread, and Poetry: Selected Prose, 1979-1985 (pp. 210-231). New York: Norton.

Rich, A. (1996). Heterosexualidad obligatoria y existencia lesbiana. DUODA Revista d'Estudis Feministes, 10, 15-45.

Rubin, G. (1996). El tráfico de mujeres: notas sobre la 'economía política' del sexo. En Lamas, M. (Comp.). El género: la construcción cultural de la diferencia sexual (pp. 35-96). México: Universidad Nacional Autónoma de México-Programa Universitario de Estudios de Género/M.Á. Porrúa.

Sandoval, C. (1995). Nuevas ciencias. Feminismo cyborg y la metodología de los oprimidos. En Otras inapropiables. Feminismos desde las fronteras(pp. 81106). Madrid: Traficantes de sueños.

Shapin, S., y Schaffer, S. (1985). Leviathan and the air pump. Hobbes, Boyle and the experimental life. N.J.: Princeton University Press.

Smith, B. (1983). Introduction. En Barbara Smith (Ed.) Home Girls: A Black Feminist Anthology (pp. xix-xii). Nueva York: Kitchen Table/Women of Color Press.

Stoller, R. (1968). Sex and Gender: On the Development of Masculinity and Femininity. New York: Science House. 
Suárez-Navas, L. y Hernández, A. (2008). Introducción. En Suárez-Navas, L. y Hernández, A (Eds.). Descolonizando el feminismo: Teorías y prácticas desde los márgenes(pp. 6-23). Madrid: Cátedra.

Trujillo, G. (2009). Del sujeto político la Mujer a la agencia de las (otras) mujeres: el impacto de la crítica queer en el feminismo del Estado español. Politica $y$ Sociedad, 46 (1-2), 161-172.

Viveros, M., Rivera, C. y Rodríguez, M. (2006). De mujeres, hombres y otras ficciones: género y sexualidad en América Latina. Bogotá: Universidad Nacional de Colombia, Tercer Mundo.

Wernerk, J. (2005). De Ialodés y Feministas. Reflexiones sobre la acción de las mujeres negras en América Latina y El Caribe. Nouvelles Questions Feministas. Feminismos Disidentes en América Latina y El Caribe, 24 (7), 27-40.

Wittig, M. (2006), El pensamiento heterosexual y otros ensayos. Madrid: Egales.

\section{Cómo citar este artículo}

Cabrera, M. y Vargas, L. (2014). Transfeminismo, decolonialidad y el asunto del conocimiento: inflexiones de los feminismos disidentes contemporáneos. Universitas Humanistica, 78, 19-37. http://dx.doi.org/10.11144/Javeriana. UH78.tdac 